Reprod. Nutr. Dévelop., 1987, 27 (2 B), 513-520.

\title{
Régulation des récepteurs et mécanismes d'action de l'hormone de croissance
}

\author{
Marie-Catherine POSTEL-VINAY, Christine KAYSER, \\ Françoise BULLIER-PICARD, J.P. CLOT
}

INSERM Unité 30, Hôpital des Enfants-Malades, 149, rue de Sèvres, 75743 Paris Cedex 15.

\section{Summary. Regulation of the receptors and mechanisms of growth hormone action.}

The initial step in the action of growth hormone (GH) is interaction with specific sites localized in target cell membranes. Growth hormone receptors have been demonstrated in different tissues and have been extensively studied in the liver.

${ }^{125}$-human growth hormone injected IV in the intact male rat is internalized in the hepatocytes by a receptor-mediated process; the radioactive material is sequentially associated with plasma membranes, endocytic structures and lysosomes.

A dramatic decrease in the number of hepatic growth hormone receptors has been demonstrated in several situations of growth defect in the rat. $\mathrm{GH}$, insulin and estrogens play a role in the regulation of hepatic growth hormone receptors, but their mechanism of action is not clear. GH receptors have been partially purified; the binding subunit has an approximate $\mathrm{MW}$ oi 110,000 in rat hepatocytes and adipocytes and in IM-9 lymphocytes, a MW of 50 to 70,000 in rabbit liver. Monoclonal antibodies raised against the $\mathrm{GH}$ receptor will facilitate studies of receptor structure and function. The postreceptor events in growth hormone action are unknown. No second messenger or mediator has been demonstrated. Elucidation of the early events that follow activation of the growth hormone receptor is a priority.

La première étape de l'action d'une hormone polypeptidique comme l'hormone de croissance $(\mathrm{GH})$ est la liaison à des sites spécifiques localisés dans la membrane des cellules cibles ; c'est le début d'une série d'événements qui permettent à l'hormone d'agir sur le métabolisme cellulaire. Si notre connaissance des récepteurs de la $\mathrm{GH}$ a un peu progressé, pour les événements qui suivent la liaison de l'hormone à son récepteur notre ignorance est grande. Parmi les difficultés qui peuvent expliquer la lenteur de notre progression, la principale reste l'identification d'une réponse in vitro à la $\mathrm{GH}$. De plus, certaines actions de la $\mathrm{GH}$, au moins l'action de croissance, sont indirectes, prises en relais par les IGFs ou somatomédines.

Des sites spécifiques de liaison pour la GH ont été décrits dans plusieurs tissus chez les mammifères (tableau). Le foie reste le tissu le plus étudié parce que des effets de la $\mathrm{GH}$ y ont été montrés depuis longtemps ; plus récemment, la démonstration de la production d'IGF par le foie, sous le contrôle de la $\mathrm{GH}$, a orienté les travaux vers la cellule hépatique. La taille du tissu hépatique permet le 
fractionnement subcellulaire et donc les études de liaison, de distribution des récepteurs.

TABLEAU

Récepteurs de l'hormone de croissance.

\begin{tabular}{|c|c|c|}
\hline Tissu & Espèce & Auteurs \\
\hline Foie & $\begin{array}{l}\text { Rat } \\
\text { Lapin } \\
\text { Souris } \\
\text { Mouton } \\
\text { Homme }\end{array}$ & $\begin{array}{l}\text { Posner et al., } 1974 \\
\text { Postel-Vinay, } 1976 \\
\text { Tsushima et Friesen, } 1973 \\
\text { Kelly et al., } 1974 \\
\text { Posner, } 1976 \\
\text { Chan, Robertson et Friesen, } 1978 \\
\text { Carr et Friesen, } 1976\end{array}$ \\
\hline Adipocytes & $\begin{array}{l}\text { Rat } \\
\text { Mouton }\end{array}$ & $\begin{array}{l}\text { Fagin et al., } 1980 \\
\text { Chan, Robertson et Friesen, } 1978\end{array}$ \\
\hline Fibroblastes en culture & $\begin{array}{l}\text { Souris } \\
\text { Homme }\end{array}$ & $\begin{array}{l}\text { Murphy, Vrhosek et Lazarus, } 1983 a \\
\text { Murphy, Vrhosek et Lazarus, } 1983 b\end{array}$ \\
\hline Lymphocytes en culture & Homme & Lesniak et al., 1974 \\
\hline Thymocytes & $\begin{array}{l}\text { Vache } \\
\text { Souris }\end{array}$ & $\begin{array}{l}\text { Arrenbrecht, } 1974 \\
i d .\end{array}$ \\
\hline Ovaire & Mouton & Chan, Robertson et Friesen, 1978 \\
\hline
\end{tabular}

\section{Devenir de l'hormone dans une cellule cible}

Nous avons examiné le devenir de l'hGH in vivo, chez le rat mâle, à l'aide de techniques de fractionnement subcellulaire. ${ }^{125} \mathrm{I}-\mathrm{hGH}$ est injectée par voie IV et la distribution subcellulaire de la radioactivité est examinée en fonction du temps (Postel-Vinay, Kayser et Desbuquois, 1982). Un groupe de rats reçoit I'125/-hGH seulement, alors qu'un autre groupe reçoit la même quantité de ligand marqué avec un excès $(100 \mu \mathrm{g})$ d'hormone native : la liaison totale et la liaison non spécifique peuvent être calculées. Le maximum de fixation est trouvé dans I'homogénat hépatique 15 minutes après l'injection. Dans les cinq premières minutes, la radioactivité est associée principalement aux membranes plasmiques, de densité élevée ; puis la radioactivité est retrouvée dans des membranes de densité plus faible sédimentant avec les fractions de Golgi : le maximum de marquage de ces fractions est à 15 minutes après l'injection avec une liaison spécifique de 2,5\% de la radioactivité injectée, par $\mathrm{mg}$ de protéines de la fraction Golgi intermédiaire. Pour préciser la nature des éléments intracellulaires auxquels la radioactivité est associée, des fractions enrichies en endosomes ont été préparées à l'aide de deux gradients successifs : gradients continus de saccharose et de Nycodenz (*) (Kindberg et al., 1984). Dans les fractions du gradient de Nycodenz le pic de radioacti-

(*) Nycodenz : dérivé triiodé de l'acide benzoïque. 
vité est retrouvé à une densité de 1,096 ; il est distinct du maximum d'activité de la galactosyltransférase (densité 1,116). ${ }^{125} \mathrm{I}$-hGH injectée à I'animal intact entre donc dans l'hépatocyte en s'associant d'abord aux membranes plasmiques puis à des structures endosomales physiquement séparables des éléments du Golgi.

D'autres études utilisant des techniques morphologiques indiquent aussi que la GH pénètre dans les cellules : dans des lymphocytes humains en culture, 125|hGH est retrouvée à l'intérieur de la cellule dans des structures possédant les caractéristiques des lysosomes (Barazzone et al., 1980).

Comme les autres hormones polypeptidiques, la $\mathrm{GH}$ couplée à son récepteur entre donc dans les cellules cibles; les molécules de ligand se dissocient ensuite des récepteurs et sont dégradées dans les lysosomes, alors qu'une partie au moins des récepteurs va regagner la surface de la cellule; ces différentes étapes ne sont pas clairement établies pour l'hormone de croissance.

\section{Spécificité des récepteurs de l'hormone de croissance}

Seule I'hormone de croissance de primates est active chez l'homme et cette spécificité d'espèce est contrôlée au niveau du récepteur : les récepteurs de tissus humains ne reconnaissent pas les molécules de GH autres que l'hGH (Lesniak et al., 1974 ; Carr et Friesen, 1976). L'utilisation de ligands hétérologues doit être précédée d'une étude attentive du modèle étudié, notamment pour l'interprétation de résultats d'un " radioreceptor assay ". ${ }^{125} \mathrm{I}-\mathrm{hGH}$ est le ligand le plus utilisé pour suivre les récepteurs de la $\mathrm{GH}$; or cette hormone possède une activité de croissance et aussi une activité lactogénique, ce qui est reflété au niveau des récepteurs : I'hGH partage avec les autres GH des sites " somatotropiques " et avec les hormones lactogéniques des sites «lactogéniques". Dans les membranes hépatiques de rat femelle après la puberté, les sites lactogéniques prédominent en nombre ; par contre dans le foie de rat mâle, on ne décèle que des sites « somatotropiques " (Postel-Vinay, 1976).

\section{Régulation des récepteurs}

La réponse d'un tissu cible à une hormone est modulée par le taux plasmatique de l'hormone mais aussi par la disponibilité des récepteurs avec lesquels le ligand peut interagir. Nous avons donc examiné les récepteurs hépatiques de la GH dans des situations de défaut de croissance chez le rat. Dans l'insuffisance rénale chronique (Finidori, Postel-Vinay et Kleinknecht, 1980), dans le jeûne (Postel-Vinay, Cohen-Tanugi et Charrier, 1982), dans le diabète induit par la streptozotocine (Baxter, Bryson et Turtle, 1980) une importante diminution du nombre des récepteurs hépatiques de la GH a été démontrée. Ces changements sont rapides et importants : par exemple, après 4 jours de jeûne, la liaison de la bGH (GH bovine) est de $50 \%$ de la liaison aux membranes hépatiques des rats témoins ; il s'agit d'une diminution du nombre des récepteurs, sans altération significative de l'affinité de la liaison. Tous les compartiments de récepteurs de la cellule hépatique sont atteints : la diminution de l'activité de liaison est la même dans les membranes 
plasmiques et les fractions de Golgi. La réparation est également rapide : chez les rats réalimentés pendant 3 jours, les récepteurs de la $\mathrm{GH}$ sont comparables aux témoins.

Dans ce modèle du rat à jeun/réalimenté, nous avons pu mettre en évidence une corrélation positive entre la liaison de la bGH aux membranes microsomales de foie et l'activité somatomédine plasmatique (Postel-Vinay, Cohen-Tanugi et Charrier, 1982). Ces résultats suggèrent que la GH contrôle l'activité somatomédine produite par le foie à travers ses récepteurs ; ils indiquent aussi l'importance physiologique des changements dans la concentration des récepteurs hépatiques. Cependant nous sommes toujours à la recherche d'un effet plus précoce de l'hormone de croissance dans l'hépatocyte que nous pourrions mesurer en même temps que les récepteurs. Un des seuls exemples où la liaison de la $\mathrm{GH}$ a pu être corrélée avec des effets métaboliques de l'hormone est un travail sur les adipocytes de rats (Grichting, Levy et Goodman, 1983) : dans des cellules capables de réponses de type insulinique à la $\mathrm{GH}$, des récepteurs à spécificité somatotropique ont été mis en évidence.

\section{Facteurs régulateurs des récepteurs}

L'ontogenèse des récepteurs hépatiques de la $\mathrm{GH}$ a été étudiée chez le rat: la liaison de la bGH est augmentée deux fois chez la femelle au moment de la puberté, alors que chez le mâle la liaison de la bGH est constante avant et après la puberté (Postel-Vinay, 1976). La gestation s'accompagne d'une augmentation de deux fois de la liaison de la bGH aux membranes microsomales de foie chez la lapine (Kelly et al., 1974). L'œstradiol a donc certainement un rôle dans la régulation des récepteurs hépatiques de la $\mathrm{GH}$.

Le rôle de la $\mathrm{GH}$ elle-même n'est pas très clair : dans des situations opposées en ce qui concerne les taux circulants de $\mathrm{GH}$, chez le rat hypophysectomisé (Picard et Postel-Vinay, 1984) et chez le rat porteur d'une tumeur sécrétant de la $\mathrm{GH}$ (Baxter, Zoltsman et Turtle, 1982), une augmentation du nombre des récepteurs hépatiques de la $\mathrm{GH}$ a été retrouvée. Rappelons que dans ces situations où les taux plasmatiques de GH sont élevés, les membranes sont traitées de façon à les désaturer de l'hormone endogène et à mesurer les récepteurs " totaux ».

Dans le jeûne et le diabète induit par la streptozotocine, à une sécrétion basse de $\mathrm{GH}$ correspond une diminution des récepteurs hépatiques de la $\mathrm{GH}$. D'autre part les injections de $\mathrm{GH}$ pendant plusieurs jours induisent peut-être des récepteurs hépatiques mais les résultats doivent être interprétés prudemment : l'apparition rapide d'anticorps dirigés contre la $\mathrm{GH}$ pourrait simuler une liaison à des récepteurs (Hughes, Elsholtz et Friesen, 1982).

II faut aussi distinguer un éventuel rôle à long terme de la GH sur l'induction de son récepteur et un effet rapide de "down-regulation ». Dans les heures qui suivent l'exposition de lymphocytes en culture à la $\mathrm{GH}$, le nombre des récepteurs diminue de façon importante. Dans les heures qui suivent l'injection IV de GH à des rats mâles, une diminution de la liaison de la $\mathrm{GH}$ aux membranes hépatiques est retrouvée (Postel-Vinay, résultats non publiés). 
Des arguments existent en faveur d'un rôle pour l'insuline dans la régulation des récepteurs de la GH. Baxter et al. (1980) ont montré que les récepteurs de la $\mathrm{GH}$, très diminués dans le diabète induit par la streptozotocine, sont partiellement restaurés après traitement des rats par l'insuline. De même dans le modèle jeûne/réalimentation, la liaison de la $\mathrm{GH}$ aux membranes hépatiques suit les variations de l'insulinémie.

L'étude des récepteurs de la $\mathrm{GH}$ chez le rat Zucker fa/fa qui présente une obésité génétique est en cours dans le laboratoire. L'insulinémie chez le rat obèse augmente vers le $18^{e}$ jour de vie : elle est significativement plus élevée chez les rats obèses de 20 jours que chez les rats minces des mêmes portées. Nous avons pu montrer une augmentation de la liaison de la $\mathrm{GH}$ aux membranes plasmiques et aux fractions de Golgi de rats obèses à partir de la quatrième semaine de vie. L'augmentation du nombre de récepteurs apparaît donc après l'installation de I'hyperinsulinémie chez les animaux obèses. Ces résultats apportent de nouveaux arguments en faveur d'un rôle de l'insuline dans la régulation des récepteurs hépatiques de la GH (Postel-Vinay et al., 1985).

La GH, les œestrogènes et l'insuline ont donc un rôle régulateur mais il n'est pas encore possible de déterminer si ces hormones fonctionnent de façon indépendante ou de façon indirecte, par l'intermédiaire d'autres hormones.

Une question peu étudiée est de savoir si la régulation des récepteurs d'une hormone polypeptidique existe en même temps dans les différents tissus cibles de l'hormone; alors que les récepteurs hépatiques de la $\mathrm{GH}$ sont augmentés en nombre, la liaison de la $\mathrm{GH}$ aux adipocytes est diminuée chez le rat hypophysectomisé.

\section{Mécanismes de régulation des récepteurs}

Les différents travaux ont plus porté sur le résultat de la régulation, c'est-àdire une augmentation ou une diminution des récepteurs, que sur les mécanismes de la régulation des récepteurs. Le principal mécanisme est certainement un changement de vitesse de synthèse des récepteurs; mais d'autres mécanismes de régulation peuvent s'ajouter : l'activation de récepteurs cryptiques, la stimulation d'enzymes responsables de l'internalisation ou de la dégradation des récepteurs, par exemple.

Les résultats de la liaison de la $\mathrm{GH}$ à ses récepteurs doivent être interprétés en fonction des taux plasmatiques de GH. En effet, des changements dans les concentrations plasmatiques affectent la proportion de sites " occupés " et de sites " libres ». La dissociation de la $\mathrm{GH}$ de son récepteur est un phénomène lent, si bien qu'un taux plasmatique élevé de $\mathrm{GH}$ peut simuler une diminution de l'activité de liaison des membranes plasmiques. II faut alors, pour évaluer les récepteurs totaux, utiliser une technique de désaturation des sites : le chlorure de magnésium à forte molarité $\left(\mathrm{MgCl}_{2} 4 \mathrm{M}\right)$ permet la dissociation hormone-récepteur en laissant aux récepteurs leur fonction de liaison (Kelly, Leblanc et Djiane, 1979).

Nous savons peu de choses sur la biosynthèse des récepteurs hépatiques de la $\mathrm{GH}$. Une synthèse protéique normale est nécessaire pour maintenir la concen- 
tration des récepteurs de la $\mathrm{GH}$ dans le foie de rat : le traitement de rats par la cycloheximide entraîne très rapidement une disparition des récepteurs de la $\mathrm{GH}$, avec une demi-vie du récepteur estimée à 30 min environ (Baxter, 1985). Mais ces données n'apportent pas la preuve que le récepteur est synthétisé de novo; d'autres protéines régulatrices pourraient être en cause. On sait qu'il existe une forte concentration de récepteurs de la $\mathrm{GH}$ dans les fractions enrichies en Golgi : 50 à $60 \%$ des récepteurs de l'hépatocyte sont distribués dans ces fractions (Picard et Postel-Vinay, 1984). Ils représentent probablement des récepteurs synthétisés attendant leur migration et leur incorporation dans les membranes plasmiques.

\section{Purification du récepteur de la GH. Structure}

Des récepteurs de la $\mathrm{GH}$ ont été solubilisés et purifiés partiellement à partir de foie de lapine (Waters et Friesen, 1979; Herington et Weith, 1977) et de lymphocytes en culture (McGuffin et al., 1976).

L'analyse par chromatographie a montré que le récepteur solubilisé par le Triton $\times 100$ est élué avec un poids moléculaire de 200 à 300000 . Mais à cause de la présence du détergent, cette technique surestime le PM. Plus récemment par des techniques de "cross-linking " le récepteur de la $\mathrm{GH}$ a été étudié dans l'hépatocyte de rat (Donner, 1983), dans l'adipocyte (Carter-Su et al., 1984) et le lymphocyte IM-9 (Hughes, Simpson et Friesen, 1983). L'analyse par électrophorèse dans des conditions de réduction montre un poids moléculaire de 108 à 112000 pour ces récepteurs avec probablement une seule chaîne. Pour le récepteur du foie de lapine dont le PM est entre 50 et 67 000, l'existence de sous-unités est probable (Hughes, Simpson et Friesen, 1983).

Le développement d'anticorps antirécepteur devrait apporter une meilleure connaissance de la fonction du récepteur. Des anticorps monoclonaux dirigés contre le récepteur de la $\mathrm{GH}$ de foie de lapine ont été préparés (Simpson, Hughes et Friesen, 1983 ; Barnard et al., 1985) et permettent la purification plus facile des récepteurs par chromatographie d'affinité.

\section{Evénements « post-récepteur 》}

Nos connaissances des événements qui surviennent après l'interaction de la $\mathrm{GH}$ avec son récepteur se réduisent à très peu. II n'y a pas de résultats indiquant une phosphorylation du récepteur ou la présence d'une protéine kinase associée au récepteur. Nous ne connaissons pas de deuxième messager pour les effets de la GH. L'activation des récepteurs par l'hormone ne semble pas entraîner de changements de l'activité de l'adénylate cyclase : les résultats sont contradictoires, montrant tantôt une augmentation, tantôt une diminution, tantôt l'absence de changement dans les taux d'AMP cyclique. Cependant un travail récent de Vesely (1981) montre que la GH stimule l'activité guanylate cyclasique in vitro dans plusieurs tissus ; le GMP cyclique pourrait être un deuxième messager pour les effets de la $\mathrm{GH}$. 
Une autre voie est apparue depuis la démonstration de la régulation de la synthèse d'ARNs messagers et de protéines par la GH. La possibilité de cloner des ARN messagers GH-dépendants est ouverte (Spiegelman, Frank et Green, 1983). Il devrait être possible d'étudier les gènes des protéines qui sont régulées par la $\mathrm{GH}$.

$12^{e}$ Réunion du groupe Développement I.N.R.A. Montpellier, 28-30 mai 1986.

\section{Références}

ARRENBRECHT S., 1974. Specific binding of growth hormone to thymocytes. Nature, 252. 255-257.

BARAZZONE P., LESNIAK M. A., GORDEN P., VAN OBBERGHEN E., CARPENTIER J. L., ORCI L., 1980 . Binding, internalization and lysosomal association of ${ }^{125}$-human growth hormone in cultured human lymphocytes : a quantitative morphological and biochemical study. J. Cell Biol., 87, 360-369.

BARNARD R., BUNDESEN P. G., BRYLATT D. B., WATERS M., 1985. Evidence from the use of monoclonal antibody probes for structural heretogeneity of the growth hormone receptor. Biochem. J., 231, 459-468.

BAXTER R. C., BRYSON J. M., TURTLE J. R., 1980. Somatogenic receptors of rat liver : regulation by insulin. Endocrinology, 107, 1176-1181.

BAXTER R. C., ZOLTSMAN Z., TURTLE J. R., 1982. Induction of somatogenic receptors in livers of hypersomatotropic rats. Endocrinology, 111, 1020-1022.

BAXTER R. C., 1985. Measurement of growth hormone and prolactin receptor turnover in rat liver. Endocrinology, 117, 650-655.

CARR D., FRIESEN H. G., 1976. Growth hormone and insulin binding to human liver. J. clin. Endocrinol. Metab., 42, 484-493.

CARTER-SU C., SCHWARTZ J., KIKUCHI G., 1984. Identification of a high affinity growth hormone receptor in rat adipocyte membranes. J. biol. Chem., 259, 1099-1104.

CHAN J. S. D., ROBERTSON H. A., FRIESEN H. G., 1978. Distribution of binding sites for ovine placental lactogen in the sheep. Endocrinology, 102, 632-640.

DONNER D., 1983. Covalent coupling of human growth hormone to its receptor on rat hepatocytes. J. biol. Chem., 258, 2736-2743.

FAGIN K. D., LACKEY S. L., REAGAN C. R., DigIROLAMO M., 1980. Specific binding of growth hormone by rat adipocytes. Endocrinology, 107, 608-615.

FINIDORI J., POSTEL-VINAY M. C., KLEINKNECHT C., 1980. Lactogenic and somatotropic binding sites in liver membranes of rats with renal insufficiency. Endocrinology, 106, 1960-1965.

GRICHTING G., LEVY L. K., GOODMAN H. M., 1983. Relationship between binding and biological effects of human growth hormone in rat adipocytes. Endocrinology, 113, 1111-1120.

HERINGTON A. C., VEITH N. M., 1977. Solubilization of a growth hormone specific receptor from rabbit liver. Endocrinology, 101, 984-987.

HUGHES J. P., ELSHOLTZ H. P., FRIESEN H. G., 1982. Up regulation of lactogenic receptors. An immunological artefact? Endocrinology, 111, 702-704.

HUGHES J. P., SIMPSON J. S. A., FRIESEN H. G., 1983. Analysis of growth hormone and lactogenic binding sites cross-linked to iodinated human growth hormone. Endocrinology, 112. 1980-1985.

KELLY P. A., POSNER B. I., TSUSHIMA T., FRIESEN H. G., 1974. Studies of insulin, growth hormone and prolactin binding : ontogenesis, effects of sex and pregnancy. Endocrinology, 95, $532-539$.

KELLY P. A., LEBLANC G., DJIANE J., 1979. Estimation of total prolactin binding sites after in vitro desaturation. Endocrinology, 104, 1631-1638.

KINDBERG G. M., FORD T., BLOMHOFF R., RICKWOOD D., BERG T., 1984. Separation of endocytic vesicles in Nycodenz gradients. Anal. Biochem., 142, 455-462. 
LESNIAK M., GORDEN P., ROTH J., GAVIN III J. R., 1974. Binding of 125I-human growth hormone to specific receptors in human cultured lymphocytes. J. biol. Chem., 249, $1661-1667$.

McGUFFIN W., GAVIN III J. R., LESNIAK M., GORDEN P., ROTH J., 1976. Water-soluble specific growth hormone binding sites from cultured human lymphocytes : preparation and partial characterization. Endocrinology, 98, 1661-1667.

MURPHY L. J., VRHOSEK E., LAZARUS L., 1983a. Characterization of specific growth hormone binding sites in mouse fibroblasts. Endocrinology, 113, 750-757.

MURPHY L. J., VRHOSEK E., LAZARUS L., 1983b. Identification and characterization of specific growth hormone receptors in cultured human fibroblasts. J. clin. Endocrinol. Metab., 57, 1117-1124.

PICARD F., POSTEL-VINAY M. C., 1984. Hypophysectomy and growth hormone receptors in liver membranes of male rats. Endocrinology, 114, 1328-1333.

POSNER B. I., 1976. Characterization and modulation of growth hormone and prolactin binding in mouse liver. Endocrinology, 98, 645-654.

POSNER B. I., KELLY P. A., SHIU R. P. C., FRIESEN H. G., 1974. Studies of insulin, growth hormone and prolactin binding : tissue distribution, species variation and characterization. Endocrinology, 95, 521-531.

POSTEL-VINAY M. C., 1976. Binding of human growth hormone to rat liver membranes : lactogenic and somatotropic sites. FEBS Lett., 69, 137-140.

POSTEL-VINAY M. C., COHEN-TANUGI E., CHARRIER J., 1982. Growth hormone receptors in rat liver membranes : effects of fasting and refeeding and correlation with plasma somatomedin activity. Mol. cell. Endocrinol., 28, 657-669.

POSTEL-VINAY M. C., KAYSER C., DESBUQUOIS B., 1982. Fate of injected human growth hormone in the female rat liver in vivo. Endocrinology, 111, 244-251.

POSTEL-VINAY M. C., DURAND D., LOPEZ S., LAVAU M., 1985. Growth hormone receptors and the onset of hyperinsulinemia in the obese Zucker rat. Second Joint Meeting of LWPESESPE, Baltimore June 1985 (Abst.).

SIMPSON J. S. A., HUGUES J. P., FRIESEN H. G., 1983. A monoclonal antibody to the growth hormone receptor of rabbit liver membranes. Endocrinology, 112, 2137-2141.

SPIEGELMAN B. M., FRANK M., GREEN M., 1983. Molecular cloning of mRNA from 3T3 adipocytes. J. biol. Chem., 258, 10083-10089.

TSUSHIMA T., FRIESEN H. G., 1973. Radioreceptor assay for growth hormone. J. clin. Endocrinol. Metab., 37, 334-337.

VESELY D. L., 1981. Human and rat growth hormone enhance guanylate cyclase activity. Am. J. Physiol., 240, E79-E82.

WATERS M. J., FRIESEN H. G., 1979. Purification and partial characterization of a non primate growth hormone receptor. J. biol. Chem., 254, 6815-6825. 